\title{
Estado neurológico e cardiorrespiratório de filhotes de cães nascidos de parto normal ou de cesariana sob anestesia geral inalatória com sevofluorano
}

\author{
Neurological and cardiocirculatory investigation of dog neonates born by normal parturition or \\ cesarean section on sevoflurane inhalation anesthesia
}

\author{
Daniela Tozadore Gabas $^{1}$ Lidia Mitsuko Matsubara $^{1}$ Valéria Nobre Leal de Souza Oliva ${ }^{2}$ \\ Leandro Rodello ${ }^{3}$ Cláudio Nazaretian Rossi ${ }^{4}$ Silvia Helena Venturolli Perri ${ }^{5}$
}

\section{RESUMO}

A anestesia obstétrica possibilita um procedimento mais seguro para a mãe e para os fetos. Em medicina veterinária, no entanto, a literatura científica a respeito do assunto é deficiente. Este trabalho teve como objetivo avaliar o grau de depressão neurológica, hemodinâmica e respiratória fetais provocado pelo agente anestésico, em que as mães foram submetidas ao parto normal ou à cesariana, utilizando-se sevofluorano como agente de manutenção anestésica, comparando-o com o parto normal. Foram realizados seis partos normais (GN) e seis cesarianas (GC), avaliando-se um total de 36 filhotes. As cesarianas foram realizadas utilizandose acepromazina, propofol e sevofluorano (GC) e os neonatos foram avaliados clinicamente ao primeiro, quinto e décimo minuto de nascimento, nos dois grupos. Observou-se maior depressão respiratória nos filhotes nascidos de cesariana. Contudo, apesar dessa depressão, o protocolo anestésico empregado não comprometeu de maneira importante a viabilidade e a saúde das mães e dos filhotes, demonstrando ser seguro em cadelas gestantes.

Palavras-chave: cão, parto normal, cesariana, neonato, anestesia.

\section{ABSTRACT}

The obstetric anesthesia must be safe for mother and puppies and about this, the literature is pour. This study was aimed at evaluating the neurological, hemodinamic and respiratory changes in neonates provoked by the anestesic agent as a result of normal parturition and cesarean section employing sevoflurane as the maintenance agent. Six deliveries (GN) and

\begin{abstract}
six cesarean sections (GC) were performed. The cesarean sections were performed under general anesthesia using acepromazina maleate, propofol and sevoflurane. Thirty six puppies were evaluated and the neurologic reflexes were worse in that were born through cesarean section. However, we concluded that despite the anesthetic depression, the protocol employed didn't affect in any important way the viability and health of the mothers and puppies, being suitable for cesarean sections.
\end{abstract}

Key words: dog, normal parturition, cesarean section, neonate, anaesthesia.

\section{INTRODUÇÃO}

Em medicina veterinária, nota-se a necessidade de maiores estudos referentes aos procedimentos anestésicos em gestantes, pois as informações a respeito da anestesia nesses pacientes e de seus efeitos nos fetos são escassas. Devido às propriedades físico-químicas dos fármacos, é impossível que eles consigam atravessar a barreira hematoencefálica materna sem penetrar na barreira placentária (THURMON et al., 1996). Dessa forma, o grau de depressão fetal é diretamente proporcional ao grau de depressão materna (DE BIASI, 1999) e os efeitos dos anestésicos atingem o feto diretamente, por cruzarem a placenta, ou indiretamente, por alterarem a função cardiopulmonar materna.

${ }^{1}$ Curso de Pós-graduação em Anestesiologia, Universidade Estadual Paulista (Unesp), Faculdade de Medicina de Botucatu, São Paulo, SP, Brasil. E-mail: danigabas@hotmail.com.

${ }^{2}$ Curso de Medicina Veterinária, Departamento de Clínica, Cirurgia e Reprodução Animal, Unesp, Araçatuba. Rua Clóvis Pestana, 793, Jardim D. Amélia, 16050-680, Araçatuba, SP, Brasil. E-mail: voliva@fmva.unesp.br. Autor para correspondência. ${ }^{3}$ Curso de Pós-graduação em Medicina Veterinária, Faculdade de Medicina Veterinária e Zootecnia, Unesp, Botucatu, SP. ${ }^{4}$ Curso de Pós-graduação em Cirurgia Veterinária, Faculdade de Ciências Agrárias e Veterinárias, Unesp, Jaboticabal, SP. ${ }^{5}$ Curso de Medicina Veterinária, Departamento de Apoio e Produção Animal, Unesp, Araçatuba, SP, Brasil. 
As anestesias realizadas durante a gestação e no momento do parto devem ser cautelosas, pois a permeabilidade hematoencefálica fetal é maior, o que aumenta a sensibilidade a esses fármacos. Deve-se considerar ainda que a função renal do feto é imatura, dificultando a excreção dos mesmos, além do fato de que a atividade microssômica enzimática também é deficiente (MASSONE, 2003). A homeostase mãe-feto e a sobrevivência neonatal são dependentes da manutenção da circulação útero-placenta. A oxigenação uterina é diretamente proporcional à pressão de perfusão e indiretamente proporcional à resistência vascular uterina; ou seja, como na gestante anestesiada há diminuição na circulação uterina, pode haver redução na viabilidade fetal (GAIDO, 1997).

A pressão arterial fetal média diminuiu significativamente após oito minutos de anestesia com halotano, permanecendo baixa, mas não afetando a freqüência cardíaca de forma significativa (DE BIASI, 1999). A pressão arterial e a temperatura corpórea são mais baixas nos neonatos quando comparadas com as dos adultos, embora as freqüências cardíaca e respiratória sejam mais altas (MOON et al., 2001).Todos os agentes inalatórios estão sendo utilizados com sucesso para a realização de cesariana em cadelas, mas o isofluorano é associado com uma melhor taxa de sobrevivência (ROBERTSON \& MOON, 2003).

O sevofluorano, que possui baixo coeficiente de solubilidade sangüínea, produz indução e recuperação anestésicas rápidas, o que permite fácil controle da profundidade anestésica (OLIVA,1997). Assim o sevofluorano poderia ser útil em cesarianas, mas ainda fazem-se necessárias maiores investigações a respeito dos efeitos desse fármaco, sobre a cadela gestante.

O propofol e tiopental sódico têm sido utilizados na obstetrícia humana e têm a vantagem de proporcionar uma rápida indução com mínima depressão residual fetal. O propofol foi associado com um melhor vigor fetal quando comparado com o tiopental e o tiamilal (ROBERTSON \& MOON, 2003).

O estado mental dos neonatos é determinado pela resposta a estímulos externos, pela resposta de acordar subitamente quando o animal é retirado da mãe e pela qualidade do choro do neonato, podendo-se também avaliá-lo através da constatação da presença ou ausência dos reflexos de dor, de sucção, ano-genital, magno ou flexor (FEITOSA \& CIARLINI, 2001).

Rotineiramente, em obstetrícia humana, realiza-se a avaliação do estado de recém-nascido aplicando-se a tabela Apgar (Tabela 1- adaptada) no primeiro, no quinto e no décimo minuto após o nascimento (SEGRE \& ARMELLINI, 1981), sendo a avaliação do sistema nervoso um tópico muito importante. DODMAN (1979) constatou valores melhores de APGAR (escala de avaliação neurológica em recém-nascidos) de crianças nascidas de cesariana eletiva quando foi administrado $66 \%$ de oxigênio no período pré-parto, mas estes dados não foram confirmados em animais.

Em estudo em que 24 cadelas foram submetidas à cesariana sob quatro diferentes protocolos anestésicos, a depressão dos reflexos neurológicos foi menor após anestesia epidural, seguida pelo propofol/enflurano, tiopental sódico/ enflurano e midazolam/cetamina/enflurano (LUNA et al., 2004).

Um estudo veterinário mostrou que, na cesariana, a mortalidade de filhotes foi de $8 \%$ ao nascimento e de $13 \%$ após duas horas e que, no parto normal, a mortalidade é de 2,2\% ao nascimento e de $8 \%$ no primeiro dia. Filhotes nascidos de cesarianas recebem maiores cuidados pós-parto, tais como: administração de fontes de calor (4\%), aplicação de doxapram (34\%) e de oxigênio (15\%) (MOON et al., 2001). Ainda segundo esses autores, a causa de morte de filhotes é indeterminada em alguns casos (28\%), sendo o trauma a causa mais comum nas primeiras 24 horas (14\%). A segunda causa é a prematuridade ou a imaturidade dos filhotes (10\%).

Em um amplo estudo em cães, a xilazina foi associada com o aumento da mortalidade nos filhotes. Outros fármacos, incluindo os anti-colinérgicos, sedativos (acepromazina) e opióides, foram usados

Tabela 1 - Tabela adaptada da escala Apgar (APGAR et al., 1958) utilizada para avaliar a evolução de filhotes caninos recém-nascidos.

Parâmetros Avaliados

ESCORE

\begin{tabular}{llll} 
& zero & 1 & 2 \\
\hline Batimentos cardíacos & Ausente & Lento: <100 & $>100$ \\
Esforços respiratórios & Ausente & Lento, irregular e choro fraco & Bom, regular, choro forte, vigoroso \\
Tônus muscular & Flácido & Certa ou alguma flexão dos membros & Movimentos ativos \\
Atividade reflexa & Ausente & Careta & Tosse ou espirro, choro \\
Cor de pele & Pálido & Cianótico & Róseo \\
\hline
\end{tabular}


com sucesso e não aumentaram significativamente a taxa de mortalidade dos filhotes. Adequando a dose ao estado da mãe, a medicação pré-anestésica pode diminuir a ansiedade, prover analgesia (diminuir a dor do parto e do procedimento cirúrgico), reduzir o requerimento anestésico e proporcionar indução e recuperação anestésicas rápidas e suaves (ROBERTSON \& MOON, 2003).

Este trabalho teve o objetivo de estudar as alterações respiratórias, circulatórias e neurológicas dos fetos decorrentes da anestesia geral inalatória com sevofluorano em cadelas pré-tratadas com acepromazina e propofol, comparando-as com as que ocorrem no parto normal.

\section{MATERIAL E MÉTODOS}

Foram avaliados 36 filhotes alocados em dois grupos distintos: nascidos de parto normal (GN) ou por meio de cesariana (GC). Tanto as mães quanto os neonatos foram mantidos no canil experimental do Hospital Veterinário Luiz Quintiliano de Oliveira, do Curso de Medicina Veterinária da UNESP, Campus de Araçatuba, e, ao final do experimento, todos foram doados para a população local.

Doze partos foram realizados, sendo seis partos normais (GN) e seis cesarianas (GC), com a participação de oito cadelas diferentes, das quais quatro cadelas tiveram o nascimento dos filhotes da primeira gestação por meio de parto normal e da segunda gestação, por meio de cesariana. As demais participaram de apenas um dos grupos, sendo duas submetidas ao parto normal e duas à cesariana.

Para a determinação do momento correto de realização da cesariana eletiva e da maturidade completa dos filhotes, foram considerados os seguintes parâmetros: tempo de gestação (60 a 63 dias decorridos da primeira cobertura), evolução radiográfica (visualização da pelve fetal entre 53 e 57 dias de gestação) (FELDMAN \& NELSON, 1996) e diminuição da temperatura retal. Este último parâmetro foi aferido duas vezes ao dia a partir de uma semana antes da data prevista para o parto e, quando a temperatura retal atingia $37,5^{\circ} \mathrm{C}$ ou ocorria a redução de $1^{\circ} \mathrm{C}$ em relação à temperatura normal do animal, considerava-se que o parto deveria ter início dentro das 24 horas seguintes (ALLEN, 1995). Quando a temperatura retal atingia $37,5^{\circ} \mathrm{C}$, passava-se a aferi-la em intervalos de três horas. Para a realização da cesariana eletiva, considerou-se o momento em que a temperatura retal começava a se elevar, após o período de diminuição. Nos animais que seriam submetidos ao parto normal, deixou-se que o trabalho de parto evoluísse de maneira natural, realizando-se também os exames radiográficos (para confirmação da visualização da pelve fetal indicando a última semana de gestação), a monitoração da temperatura retal, a observação da eliminação do tampão mucoso, considerando-se ainda o tempo decorrido da cobertura.

\section{Grupo normal (GN)}

Após os primeiros sinais clínicos do começo do trabalho de parto, da elevação da temperatura, das primeiras contrações e da expulsão do tampão mucoso, realizou-se o exame de ultra-sonografia a abdominal, com transdutor transabdominal de $5 \mathrm{mHz}$, dividindo-se o abdômen em quatro quadrantes e procurando-se obter a freqüência cardíaca de pelo menos um feto em cada quadrante, realizando-se então a média aritmética desses valores, obtendo-se a média da freqüência cardíaca fetal (FCf) (M1), antes do primeiro nascimento. Para efeito comparativo, após um minuto do primeiro, terceiro e último filhote, foi realizada nova mensuração deste parâmetro por meio da contagem direta, por auscultação com estetoscópio (M2), tanto naqueles nascidos por cesariana quanto por parto normal. A média aritmética destes valores corresponde a FCf.

\section{Grupo cesariana (GC)}

As cadelas deste grupo foram submetidas à fluidoterapia com Ringer com lactato na taxa de infusão de $10 \mathrm{ml} \mathrm{dg}^{-1} \mathrm{~h}^{-1}$ e à medicação pré-anestésica, que foi realizada com acepromazina $0,2 \%{ }^{\mathrm{b}}\left(0,05 \mathrm{mg} \mathrm{kg}^{-1}\right.$, via intravenosa, bolus). Como agente indutor, utilizou-se o propofolc (5 mg kg-1, via intravenosa, bolus em um minuto). A manutenção anestésica foi feita com sevofluorano ${ }^{\mathrm{d}}$ na concentração necessária para manter o plano II do $3^{\circ}$ estágio anestésico (MASSONE, 2003). O exame de ultra-sonografia foi realizado da mesma maneira descrita para o parto normal, sendo M1 realizado antes da medicação pré-anestésica do neonato. Durante a manutenção anestésica, foi realizada a operação cesariana e a anestesia foi mantida por 60 minutos, ainda que a cirurgia terminasse antes.

Nos recém-nascidos, realizaram-se, em ambos os grupos, as avaliações de maturidade por meio da escala Apgar adaptada da escala de uso humano, no $1^{\circ}$, $5^{\circ}$ e $10^{\circ}$ minuto de vida do neonato (Tabela 1 ) e do grau de depressão neurológica por meio de resposta positiva ou ausente aos estímulos de sucção, anogenital, magno, dor, flexão, conforme descrito por FEITOSA \& CIARLINI (2001).

Os dados foram submetidos à análise de variância, com as médias sendo comparadas por meio do teste de Tukey. Os valores da escala de APGAR et al. (1958) das variáveis freqüência cardíaca e respiratória, 
tônus muscular e atividade reflexa foram analisados pelo teste de Mann-Whitney, para comparar os grupos em cada momento, e pelo teste de Friedman, para comparar os momentos em cada grupo. As demais variáveis desta escala e a avaliação dos reflexos neurológicos foram analisadas pelo teste de Quiquadrado ou teste exato de Fisher para verificar a associação entre as variáveis e grupos em cada momento e entre variáveis e momentos para cada grupo. As estatísticas foram consideradas significativas quando $\mathrm{P}<0,05$ e utilizou-se o programa computacional SAS para a realização das análises (SAS INTITUTE, 1997).

\section{RESULTADOS E DISCUSSÃO}

A acepromazina utilizada na medicação préanestésica nas cadelas submetidas à cesariana teve a finalidade de tranqüilizar as cadelas e potencializar a anestesia, não parecendo haver contra-indicação do seu uso em animais gestantes, uma vez que ROBERTSON \& MOON (2003) não constataram aumento significativo da taxa de mortalidade dos filhotes com o uso desta medicação.

A aplicação lenta do propofol permitiu a obtenção de plano anestésico adequado para a intubação traqueal, sendo mais apropriado para a indução de anestesia geral nas cesarianas por apresentar metabolização mais rápida do que os barbitúricos, associado ao melhor vigor fetal (ROBERTSON \& MOON, 2003).
O sevofluorano foi utilizado como agente inalatório para a manutenção anestésica por cruzar rapidamente a barreira placentária, atingindo o equilíbrio materno-fetal e, principalmente, devido ao seu baixo coeficiente de solubilidade e rápida eliminação (OLIVA, 1997). Todos os fármacos utilizados permitiram a anestesia balanceada, com estabilidade dos parâmetros e recuperação anestésica rápida e livre de excitação.

No grupo GC, as freqüências cardíacas fetais (FCf) antes do nascimento foram maiores em relação à aferição após um minuto de nascimento, e também menor neste momento, quando comparada com o mesmo momento no grupo GN (Figura 1). Isto pode ter ocorrido devido às alterações cardiocirculatórias da mãe em decorrência da anestesia. Mas também é sabido que a exposição fetal aos anestésicos, quando prolongada, pode levar à depressão respiratória e à cardiovascular (GAIDO, 1997). Enquanto isso, no parto normal, as médias dos valores da FCf não se alteraram durante os momentos e mantiveram-se um pouco abaixo dos parâmetros fisiológicos citados por MOON et al. (2001). Isso ocorreu, certamente, porque neste grupo nenhum fármaco foi administrado.

Em relação à avaliação pela escala APGAR, não foram encontradas diferenças significativas entre os grupos ou entre os momentos dentro de cada grupo em relação à FC e ao esforço respiratório, sendo atribuído o escore mais elevado (escore 2) para a maioria dos neonatos. A avaliação da cor de pele nos neonatos nascidos de cesariana apresentou diferença significativa em relação aos animais nascidos de parto normal no primeiro minuto de vida, pois foi evidenciada

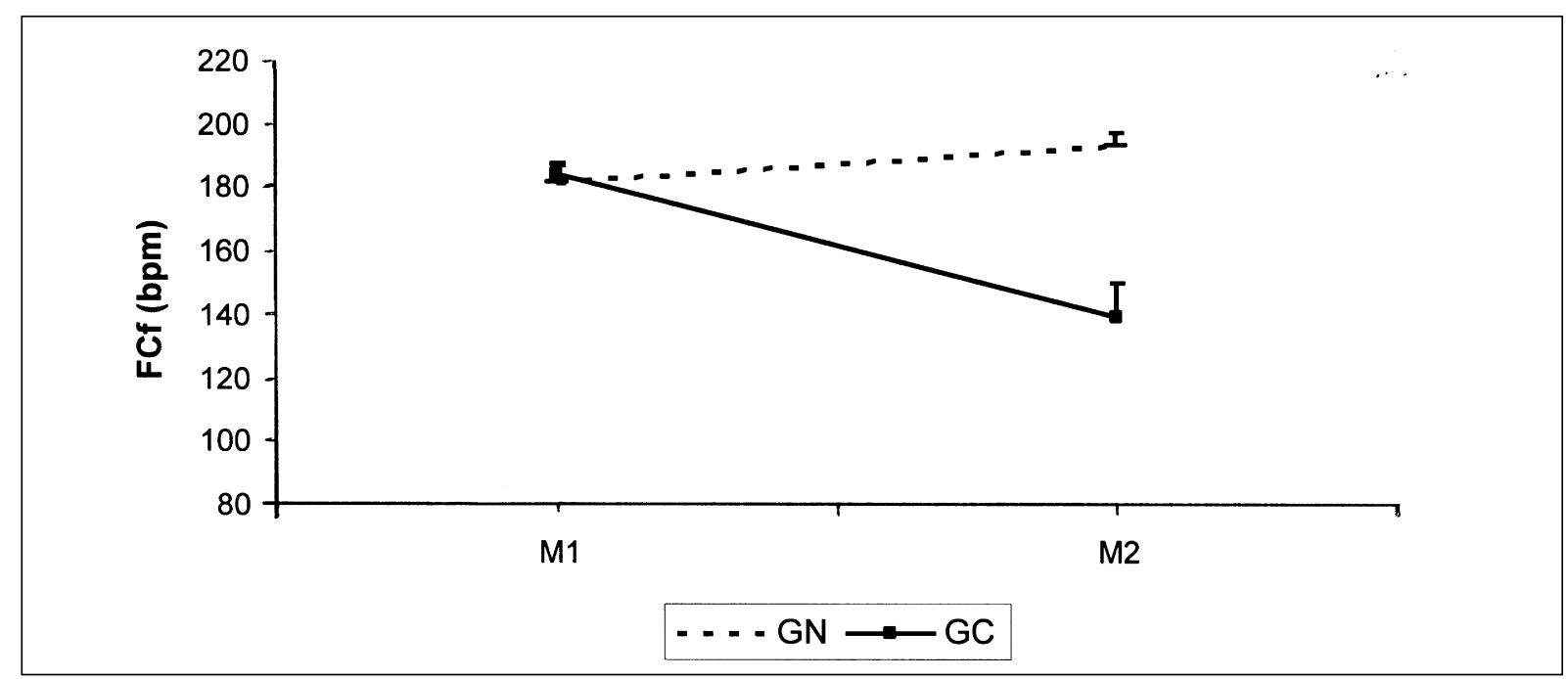

Figura 1- Médias da freqüência cardíaca fetal, em batimentos por minuto (bpm), de filhotes das cadelas nascidos de parto normal (GN) ou de cesariana (GC), realizada sob anestesia com acepromazina, propofol e sevofluorano, nos momentos M1 (antes do parto ou da anestesia) e M2 (logo após o nascimento).

Ciência Rural, v.36, n.5, set-out, 2006. 
apnéia logo após o nascimento da maioria dos filhotes nascidos de cesariana, ao contrário do que ocorreu naqueles nascidos de parto normal. Essa apnéia deveuse, provavelmente, à depressão neurológica causada pelos agentes de manutenção. A partir do quinto minuto após o nascimento, os neonatos de ambos os grupos apresentaram escore 2 (coloração rósea). Com referência à atividade reflexa e ao tono muscular, verificou-se diferença significativa entre os dois grupos em todos os momentos de avaliação, observando-se que, nos neonatos nascidos de cesariana, estas respostas estiveram ausentes até o último momento de observação (10 minutos), enquanto que, nos nascidos de parto normal, as respostas estavam presentes já no primeiro minuto.

No que se refere à avaliação neurológica, observou-se que a resposta à dor e a presença do reflexo flexor estiveram presentes já no primeiro minuto de vida em todos os filhotes nascidos de parto normal. Contudo, naqueles nascidos de cesariana, ocorreu ausência destas respostas em 33\% ao nascimento, ocorrendo elevação deste percentual ao longo dos momentos seguintes (5 e 10 minutos, 44\% e 55\% dos animais com respostas presentes, respectivamente). Os reflexos de sucção e magno estiveram presentes na maioria dos animais do GN e ausentes em praticamente todos os animais do GC, considerando-se todos os momentos de avaliação. O reflexo ano-genital também esteve mais deprimido nos animais do GC no primeiro minuto de avaliação, passando, a partir daí, a apresentar- se em um número crescente de filhotes deste grupo. Apesar dos achados, nenhuma das respostas reflexas apresentou diferenças estatisticamente significativas entre os grupos.

Estes achados deixam claro que houve depressão nos filhotes nascidos de cesariana pelo procedimento anestésico, já que o grau de depressão fetal é diretamente proporcional ao grau de depressão materna (DE BIASI, 1999). Apesar da evidente depressão respiratória e neurológica observada nos animais nascidos de cesariana, não ocorreram mortes que possam ser atribuídas ao protocolo anestésico, pois o único natimorto apresentava tamanho significativamente reduzido em relação aos demais e estado de mumificação, indicando morte anterior ao parto.

Em relação ao número de filhotes vivos, natimortos e a taxa de mortalidade, não ocorreram diferenças significativas entre os grupos, apesar de haver ocorrido a morte de um filhote no GN após três semanas de vida (oriundo de uma prole numerosa) e três no GC (um natimorto e duas mortalidades decorrentes de traumas causados pela mãe, no dia seguinte) (Figura 2). MOON et al. (2001) relatam uma taxa de mortalidade de filhotes nascidos de cesariana de $8 \%$ ao nascimento e de $13 \%$ após duas horas, o que é superior ao encontrado no presente estudo (5,5\%). Esses autores descrevem, ainda, mortalidade de $2,2 \%$ ao nascimento e de $8 \%$ no primeiro dia, em filhotes nascidos de parto normal, enquanto que, neste trabalho,

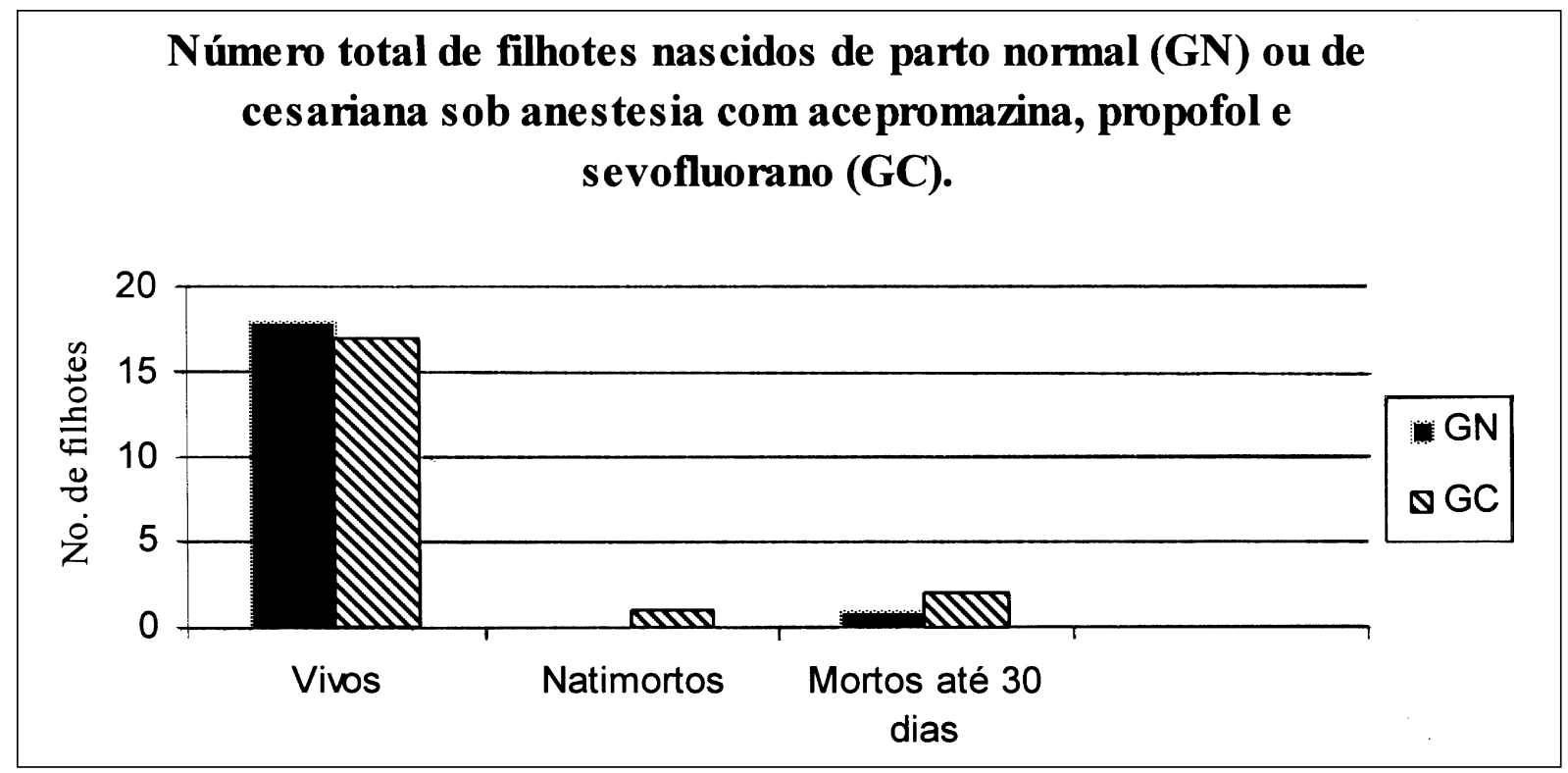

Figura 2 - Número total de filhotes nascidos de parto normal $(\mathrm{GN}, \mathrm{n}=18)$ ou de cesariana sob anestesia com acepromazina, propofol e sevofluorano (GC, $n=18$ ).

Ciência Rural, v.36, n.5, set-out, 2006. 
não ocorreram mortes neste período. Tanto nos neonatos nascidos de parto normal quanto nos de cesariana não foi realizada nenhuma manobra de ressuscitação, oxigenioterapia ou aplicação de qualquer medicamento, sendo apenas secados com compressa enquanto o examinador avaliava a escala Apgar e os reflexos neurológicos.

\section{CONCLUSÕES}

Frente aos resultados obtidos neste estudo, pode-se concluir que neonatos nascidos de cesariana não evidenciam alterações cardiocirculatórias em relação aos nascidos de parto normal. Os filhotes nascidos de cesariana têm maior depressão respiratória e depressão neurológica mais prolongada do que aqueles nascidos de parto normal. Contudo, esta depressão mais prolongada não comprometeu significativamente a viabilidade e a saúde dos filhotes. Mães e neonatos submetidos ao parto normal têm condições fisiológicas superiores em comparação aos de cesariana, imediatamente após o nascimento. Apesar disso, o protocolo anestésico utilizado neste experimento demonstrou ser seguro para a mãe e para os filhotes, podendo ser uma opção para a anestesia obstétrica.

\section{AGRADECIMENTOS}

À Fundação para o desenvolvimento da Unesp (FUNDUNESP), pelo auxílio financeiro para a realização do trabalho.

\section{COMISSÃODEÉTICA}

Este trabalho foi aprovado pela comissão de ética em experimentação animal da UNESP- Campus de Araçatuba, sob o protocolo 44/02, estando de acordo com as normas éticas do Colégio Brasileiro de Ética em Experimentação Animal (COBEA)

\section{FONTES DE AQUISIÇÃO}

a - Aparelho de ultra-som marca Aloka, modelo SSD-210 DXII, transdutor $5 \mathrm{mHz}$

b - Acepran 0,2\% - Univet S.A. Indústria Farmacêutica

c - Propofol - Cristália Produtos Químicos e Farmacêuticos Ltda.

d - Sevocris - Cristália Produtos Químicos e Farmacêuticos Ltda.

\section{REFERÊNCIAS}

ALLEN, E.W. Fertilidade e obstetrícia no cão. São Paulo: Varela, 1995. 197p.

APGAR, V. et al. Evaluation of the newborn infant-second report. J Am Med Assoc, v.168, n.15, p.1985-1988, 1958.

DE BIASI, F. Anestesia e depressão fetal. 1999. $15 f$ Seminário (apresentado à disciplina: Técnica anestésica em reprodução animal)- Faculdade de Medicina Veterinária e Zootecnia, UNESP, Campus de Botucatu.

DODMAN, N.H. Anaesthesia for caesarean section in the dog and cat: a review. J Small Anim Pract, v.20, n.8, p.449-460, 1979.

FEITOSA, M.M.; CIARLINI, L.D.R.P. Exame neurológico de cães neonatos. Cães \& Gatos, v.15, n.89, p.20-26, 2001.

FELDMAN, E.C.; NELSON, R.W. Breeding, pregnancy, and parturion. In: Canine and feline endocrinology and reproduction. 2.ed. Philadelphia: Saunders, 1996. Cap.18, p.559.

GAIDO, S.R. A gestação e a anestesia. In: ENCONTRO DE ANESTESIOLOGIA VETERINÁRIA, 3., 1997, Araçatuba. Anais... Araçatuba: Colégio Brasileiro de Cirurgia e Anestesiologia Veterinária, 1997. p.10-16

LUNA, S.P. et al. Effects of four anaesthetic protocols on the neurological and cardiorespiratory variables of puppies born by caesarean section. Vet Rec, v.154, n.13, p.387-389, 2004.

MASSONE, F. Anestesiologia veterinária: farmacologia e técnica. 4.ed. Rio de Janeiro: Guanabara Koogan, 2003. p.117, 195-198, 205-210.

MOON, P.F et al. Neonatal critical care. Vet Clin North Am: Small Anim Pract, v.31, n.2, p.343-365, 2001.

OLIVA, V.N.L.S. Anestesia inalatória em cães com uso de sevofluorano. Clínica Veterinária, v.2, n.10, p.19-22, 1997.

ROBERTSON, S.A.; MOON, P.F. Anesthetic management for cesarean section in bitches. Vet Med, v.98, n.8, p.675-696, 2003.

SAS INSTITUTE. SAS/STAT software: changes and enhancements through release 6.12. Cary, 1997. 1167p.

SEGRE, C.A.M.; ARMELLINI, P.A. RN. São Paulo: Sarvier, 1981. 44p.

THURMON, J.C et al. Anaesthesia for special patients: cesarean section patients. In:___ Lumb \& Jones' veterinary anaesthesia. 3.ed. Filadelfia: Lippincott Williams \& Wilkins, 1996. p.818-828. 